\title{
Teratoma With a Malignant Somatic Component in Pediatric Patients: The Associazione Italiana Ematologia Oncologia Pediatrica (AIEOP) Experience
}

\author{
Monica Terenziani, MD, ${ }^{1}$ Paolo $\mathrm{D}^{\prime}$ Angelo, $\mathrm{MD},{ }^{2}$ Gianni Bisogno, $\mathrm{MD}^{3}$ Renata Boldrini, $\mathrm{MD}^{4}{ }^{4}$ \\ Giovanni Cecchetto, MD, ${ }^{3}$ Paola Collini, MD, ${ }^{1}$ Massimo Conte, $\mathrm{MD}^{5}$ Tina De Laurentis, $\mathrm{MD}^{4}{ }^{4}$ Ilaria Ilari, $\mathrm{MD},{ }^{4}$ \\ Paolo Indolfi, MD, ${ }^{6}$ Alessandro Inserra, $\mathrm{MD}^{4}{ }^{4}$ Luigi Piva, $\mathrm{MD},{ }^{1}$ Fortunato Siracusa, $\mathrm{MD}^{7}$ \\ Filippo Spreafico, $\mathrm{MD}^{1}{ }^{1}$ Paolo Tamaro, $\mathrm{MD}^{8}{ }^{8}$ and Margherita Lo Curto, $\mathrm{MD}^{7}$
}

\begin{abstract}
Background. Teratoma with a malignant somatic component (TMSC) is rare but described in adults, whereas information on pediatric presentation is sparse. Procedure. The Associazione Italiana Ematologia Oncologia Pediatrica identified 14 cases of TMSC. Clinical files and pathology specimens were reviewed. Results. The series (9 female, 5 male) showed the following disease: testis (2), sacrococcygeal (3), ovary (3), retroperitoneum (3), mediastinum (2), and foot soft tissue (1). Distribution of the somatic component was: carcinoma (4), pancreatic neuroendocrine tumor (1), neuroblastoma (3), rhabdomyosarcoma (3), rhabdomyosarcoma plus liposarcoma, chondrosarcoma, neurogenic sarcoma (1), chondrosarcoma plus neuroectodermal sarcoma (1), malignant peripheral nerve sheath tumor (1). Three patients were in stage I, four in stage II, three in stage III, and four in stage IV. All but one patient underwent surgery and only females showed carcinoma components. Nine
\end{abstract}

patients relapsed or progressed and eight died. Six patients are alive and disease-free. Two patients underwent complete resection and four were treated based on transformed histologies. Relapse-free and overall survival rates were $35.7 \%$ and $42.8 \%$, respectively (median follow-up, 31 months). Conclusions. Prognosis for germ cell tumors (GCTs) containing MSC is worse than that for GCTs. The pediatric disease appears to be more heterogeneous in tumor site distribution and MSC histology than in adults. Our series suggests no effects of age, histology, or gender on outcome. Surgery has an essential role in localized disease, with complete resection highly desirable. Chemotherapy optimized for histology should include reagents directed to the somatic malignancy, if chemosensitive. Malignant GCT warrants GCT-directed chemotherapy. Pediatr Blood Cancer 2010;54:532-537. (C) 2010 Wiley-Liss, Inc.

Key words: childhood; germ cell tumors; malignant transformation; teratoma

\section{INTRODUCTION}

A malignant somatic component, either of epithelial (i.e., carcinoma) or mesenchymal (i.e., neuroblastoma or sarcoma) origin and indistinguishable from a somatic malignancy, is occasionally seen admixed with mature or immature teratoma [1-3]. This rare entity is called teratoma with a malignant somatic component (TMSC).

Germ cell tumors (GCTs) are the most common neoplasms in young men (15-35 years old), and TMSC is a rare condition, accounting for only 3-6\% of such patients. Published series report mainly males with mediastinal or testicular tumors, in which the malignant somatic component is usually a sarcoma, whereas the prevailing somatic component in ovarian TMSC (in $80 \%$ of cases) is squamous cell carcinoma [4-7]. The malignant somatic component may be present at diagnosis or only apparent at the time of analysis of residual or recurrent disease, either with or without GCT elements. The prevalence of the presence at diagnosis or at relapse is heterogeneous in published series [4,6-8].

In adults, the prognosis for GCTs that contain TMSC is worse than for GCTs in general, and the best treatment option of this rare entity remains controversial $[2,3,6,7]$. Surgery is still the standard option for localized disease. In the past, chemotherapy was considered ineffective, although cases suggesting the efficacy of chemotherapy directed towards the TMSC have been reported $[2,6]$. GCTs in childhood are rare, globally accounting for only $2 \%$ of all pediatric malignancies, but treatable with a high rate of cure. Since pediatric TMSC is very rare, the available information is sparse, no treatment guidelines have yet been developed, and cytogenetic studies are lacking [8-10].

\section{PATIENTS AND METHODS}

A retrospective analysis of the pathological and clinical files of pediatric patients who presented to the Hospital associated with the
Associazione Italiana Ematologia Oncologia Pediatrica (AIEOP) and who were treated in Italy between 1978 and 2007 identified 14 patients with TMSC. The records of these 14 patients were reviewed and their clinical characteristics and outcomes recorded. Two independent pathologists reviewed the histological material. Since patients had been classified under different staging methods or not classified at all over the lengthy time interval involved (29 years), the whole series was re-staged according to the Children's Oncology Group (COG) staging system for GCTs (Table I) [11]. Levels of alpha-fetoprotein $(\alpha \mathrm{FP})$ and beta-human choriogonadotropin $(\beta-\mathrm{HCG})$, both tumor markers for GCTs, were considered elevated when they exceeded the upper normal range or, for $\alpha \mathrm{FP}$, the upper physiological levels for age in infants younger than 1 year.

A response (complete, partial, or stable) had to persist for at least 1 month. Relapse and survival times were calculated from the date of diagnosis to the date of relapse and from diagnosis date to the latest contact for follow-up or upon death. Table II lists clinical and pathological data of the series and provides details of chemotherapy and local treatments.

${ }^{1}$ Fondazione IRCCS National Cancer Institute, Milano, Italy; ${ }^{2} \mathrm{G}$ Di Cristina Children Hospital, Palermo, Italy; ${ }^{3}$ University Hospital, Padova, Italy; ${ }^{4}$ Bambino Gesu' Research Institute, Roma, Italy; ${ }^{5}$ Gaslini Children's Hospital, Genova, Italy; ${ }^{6}$ Second University, Napoli, Italy; ${ }^{7}$ Pediatric Department, University of Palermo, Palermo, Italy; ${ }^{8}$ Burlo Garofolo Children's Hospital, Trieste, Italy

Conflict of interest: Nothing to declare.

*Correspondence to: Monica Terenziani, Pediatric Oncology Unit, Fondazione IRCCS National Cancer Institute, via Venezian 1, 20133 Milano, Italy. E-mail: monica.terenziani@istitutotumori.mi.it

Received 12 December 2008; Accepted 5 November 2009 
TABLE I. Staging of Testicular, Ovarian, and Extragonadal Tumors

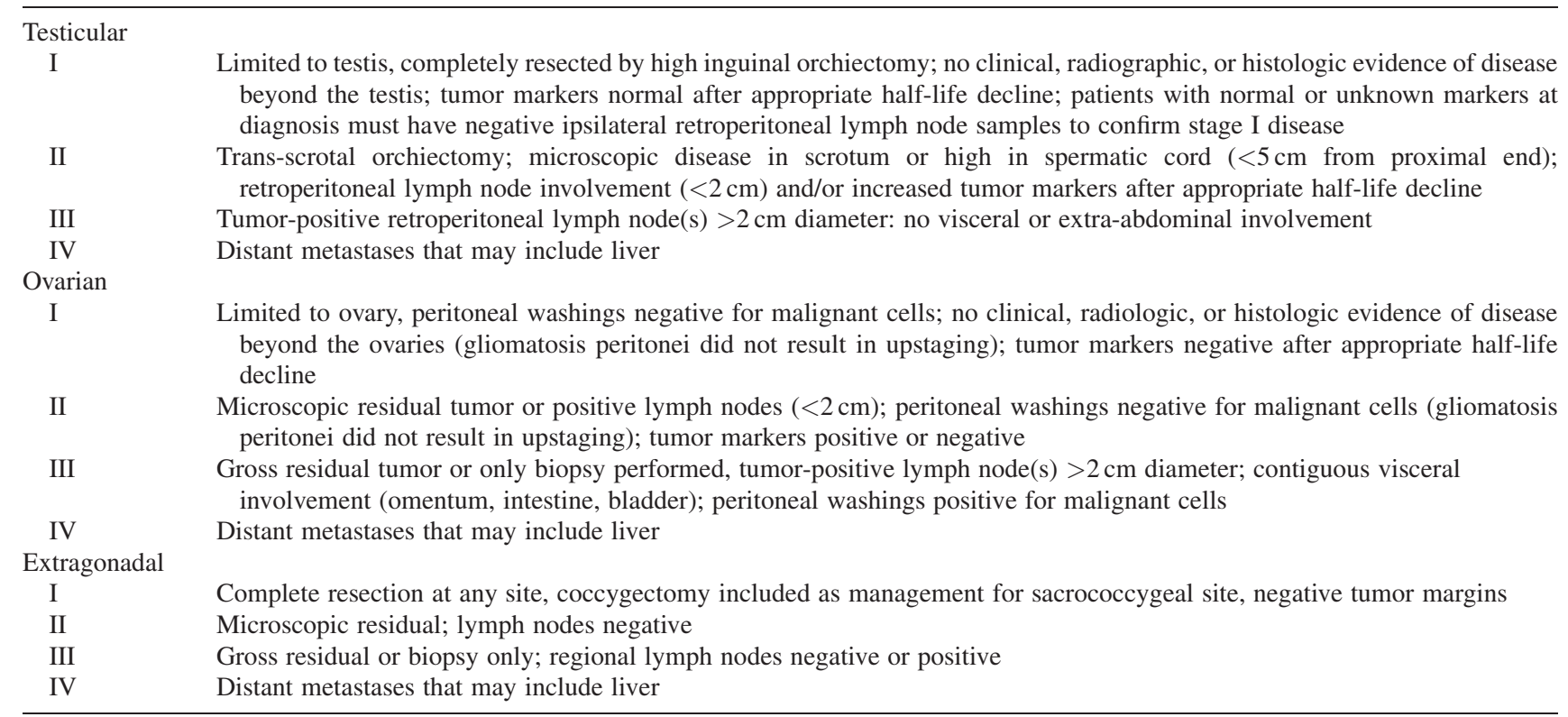

Ref. [11]

\section{RESULTS}

This series, consisting of nine females and five males with a mean age of 7.5 years at diagnosis (range 0-26 years), represents approximately the $1.4 \%$ of all extracranial pediatric GCTs observed in Italy from 1978 to 2007. Table II lists clinical and pathological data of the series and provides details of chemotherapy and local treatments.

The site of the primary tumor was: ovary (3), sacrococcygeal (3), testis (2), retroperitoneum (3), mediastinum (2), and soft tissue of the foot (1). Staging according to the COG classification system (Table I) indicated three patients in stage I, four in stage II, three in stage III, and four in stage IV. In fact, the present restaging would not have had a significant impact on the subsequent treatments and results because therapies were dictated mainly by degree of resection and type of histology. Six patients were already staged according to COG criteria, the six oldest ones were staged for the first time, and staging in two patients remained unchanged despite staging according to that in use for soft tissue sarcoma. Primary tumors were surgically resected in 11 patients at diagnosis, in 2 patients after chemotherapy, and in 1 patient, only biopsy was performed. In 11 of the 14 patients, TMSC was present at diagnosis, while the somatic component in the other 3 was detected at the time of a local relapse $(\# 2,4)$ or in the retroperitoneal lymph node and lung metastases at the time of surgery post-chemotherapy (\# 14). The reference pathologists reviewed all of the series and confirmed all diagnosis. The slides from first diagnosis and from relapse or residual tumor after chemotherapy were reviewed in four patients (\# 10,11, 13, 14), from relapse-only in two cases (\# 2, 4), and from first diagnosis in the remaining eight patients. In three cases (\#2,4,14), the MSC was not detected at diagnosis and in two of these three, slides at diagnosis were not available. In two cases $(\# 8,9)$, the percentage of YST component, which was not available at the time of diagnosis, was provided.

In 13 of 14 of these tumors, either a mature or immature teratomatous component as well as an MSC portion were observed. $\alpha \mathrm{FP}$ was elevated at diagnosis in 4 of 14 patients, accompanied by YST histology in all 4 cases, and by elevated $\beta$-HCG in 1 of these 4 . Only one male revealed chondrosarcoma and neuroectodermal sarcoma together with YST without a teratomatous component [12]. In the three cases that relapsed, there was no evidence of any increase in tumor marker levels, suggesting that the TMSC accounted for the failure of the treatment.

The TMSC was epithelial in five patients (four adenocarcinoma and one well-differentiated neuroendocrine tumor) and mesenchymal in the other nine (embryonal rhabdomyosarcoma and/or other sarcomas in five, malignant peripheral nerve sheath tumor in one, and poorly differentiated neuroblastoma in three cases). Interestingly, a carcinoma component was found in females only.

\section{Stage I Disease}

One female with mediastinal immature teratoma and welldifferentiated pancreatic neuroendocrine tumor (\# 1) had no further therapy after surgery and is disease free 18 months after diagnosis. A second 10-year-old female with retroperitoneal immature teratoma (\# 2) had no further therapy after surgery but relapsed locally 6 months later with an immature teratoma plus poorly differentiated neuroblastoma component. She was treated according to the neuroblastoma protocol with vincristine, cyclophosphamide, doxorubicin, and dactinomycin but died of disease 3 years after diagnosis. A third stage I patient (\# 3), who had an ovarian mature teratoma plus adenocarcinoma, received adjuvant chemotherapy with vincristine, dactynomicin, and cyclophosphamide (VAC regimen) according to the Italian protocol for GCTs of that time and remains disease-free 28 years after diagnosis.

\section{Stage II Disease}

A female infant (\# 4) with sacrococcygeal mature teratoma received no further treatment after surgery until the tumor recurred 


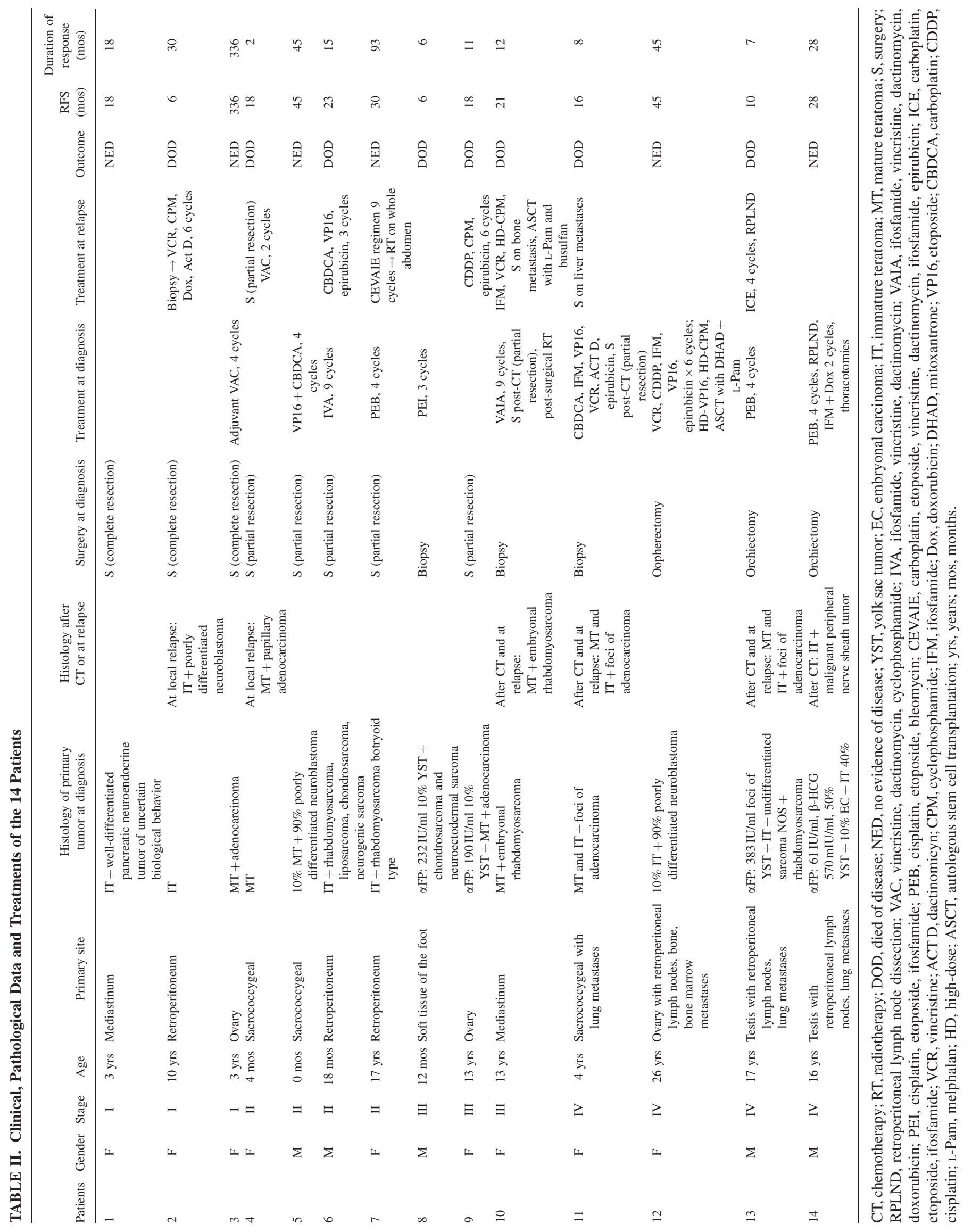


as a papillary adenocarcinoma 18 months later, when she again underwent surgery and received VAC chemotherapy; she died at 20 months after diagnosis. The other three patients (\#5-7) received systemic treatments after surgery. One male (\# 5) with congenital sacrococcygeal mature teratoma and poorly differentiated neuroblastoma received chemotherapy with carboplatin and etoposide according to the protocol for infant neuroblastoma, and remains disease-free 3.7 years after diagnosis. Patient \# 6 with retroperitoneal immature teratoma plus rhabdomyosarcoma, liposarcoma, chondrosarcoma, and neurogenic sarcoma was treated according to the rhabdomyosacroma protocol, i.e., ifosfamide, vincristine, and dactinomycin. He achieved a complete remission but relapsed locally 23 months after diagnosis and died of disease. Patient \# 7 was an adolescent with retroperitoneal immature teratoma and rhabdomyosarcoma of the botryoid type, which recurred locally 2.6 years after four cycles of cisplatin, bleomycin, and etoposide (PEB) chemotherapy. The rhabdomyosarcoma protocol was adopted as a second-line strategy, with a six-drug regimen including ifosfamide, and radiotherapy of the whole abdomen. The patient is disease-free 7.7 years after the recurrence.

\section{Stage III Disease}

A 1-year-old male (\# 8) with YST plus chondrosarcoma and neuroectodermal sarcoma in the soft tissue of the left foot underwent biopsy and chemotherapy with cisplatin, etoposide, and ifosfamide. Based on minimal shrinkage of the primary tumor, as revealed by magnetic resonance imaging, and on slow decline of $\alpha \mathrm{FP}$ levels after three cycles of chemotherapy, amputation was recommended; the parents refused the amputation and the child died of metastatic disease 12 months after diagnosis. A second patient was a 13-yearold female (\# 9) with a surgically resected ovarian cystoadenoma characterized by ascites and peritoneal implants. Given the benign histology, no further treatments were administered; however, lung, pleural, and bone metastases were detected 18 months later. At the time of relapse, a central review of histological specimens at diagnosis changed the diagnosis to YST with mature teratoma and adenocarcinoma, and the patient was therefore treated with cisplatin, epirubicin, and cyclophsphamide; however, she died of disease 11 months after relapse. The third patient (\# 10) had a mediastinal mature teratoma and embryonal rhabdomyosarcoma, which was treated according to the rhabdomyosarcoma protocol (vincristine, ifosfamide, dactinomycin, and doxorubicin), followed by surgery (with microscopic evidence of residual disease) and radiotherapy. The same histological features present at diagnosis were detected at relapse. She relapsed in the bone and, despite highdose chemotherapy, and autologous hematopoietic stem cell transplantation, died of disease 2.9 years after diagnosis.

\section{Stage IV Disease}

A young female (\# 11) with sacrococcygeal mature and immature teratoma plus adenocarcinoma with associated lung metastases received a six-drug chemotherapeutic regimen, including carboplatin, etoposide, and ifosfamide, for 10 months and achieved disease stabilization. She underwent coccygectomy, but liver metastases were detected 1 month later. Surgical resection of these metastases was attempted, but only a partial excision was feasible and she died 24 months after diagnosis. Both histology specimens showed the same features as at diagnosis. A second patient (\# 12) was a young woman with immature teratoma plus poorly differentiated neuroblastoma and bone, bone marrow, and retroperitoneal lymph node metastases. She was treated with the protocol for stage IV neuroblastoma, including cisplatin, ifosfamide, etoposide, and epirubicin, in sequential high-dose chemotherapy followed by autologous hematopoietic stem cell transplantation. The patient is alive and disease-free 3.7 years after diagnosis.

Two other cases (\# 13 and 14) were adolescent males with testicular tumors associated with retroperitoneal lymph node and lung metastases. Patient \# 13 had YST, immature teratoma, undifferentiated sarcoma, and rhabdomyosarcoma, all components already present in the primary site. Despite a normalization of $\alpha \mathrm{FP}$ after four cycles of PEB, progression of lung and retroperitoneal disease together with bone metastases were detected 1 month later. He died of disease 17 months after diagnosis. The second (\# 14) was a 16-year-old male with malignant non-seminomatous GCT of the testis without TMSC at diagnosis and with both tumor markers elevated. After four cycles of PEB, tumor marker levels returned to within normal range, and surgical excision of residual disease (lymph node and lung metastases) was planned. He underwent retroperitoneal lymph node dissection (RPLND), but histology revealed teratoma and malignant peripheral nerve sheath tumor (MPNST). After two cycles of ifosfamide and doxorubicin, the lung metastases remained stable and two subsequent thoracotomies were performed. The same histological features were detected in the resected lung metastases as in the retroperitoneal lymph nodes. The patient is disease-free 2.4 years after diagnosis.

In our series, two patients received inappropriate or incomplete treatment, with stage III patient \# 8 refusing local therapy and stage III patient \# 9 receiving no treatment except for surgery because of misdiagnosis. At the time of this report, the relapse-free and overall survival rates after a median follow-up of 31 months (range 12-336) were $35.7 \%$ and $42.8 \%$, respectively. Nine of the 14 patients relapsed or progressed, and 8 of them soon died of their disease. The median time from diagnosis to relapse was 18 months (range 6-30 months).

Six patients (two males and four females) are alive and diseasefree, with a median survival of 45 months (range 18-336 months). One female with a completely resected mediastinal tumor had received no further therapy, and only one patient who relapsed with TMSC was salvaged; the other five were cured with first-line therapy. Of the five survivors who received chemotherapy, one received adjuvant treatment directed toward GCTs, two were treated according to the neuroblastoma component, and the remaining two were treated according to GCT disease and the malignant mesenchymal component, sequentially or at relapse.

\section{DISCUSSION}

Our series of 14 patients with TMSC is representative of the disease as a whole, including all sites of presentation (gonadal and extragonadal), both genders, and different histologies (epithelial and mesenchymal), age and stage. As first-line therapy, 9 of the 14 patients were treated with surgery and chemotherapy, 4 underwent surgery as unique treatment, and 1 received chemotherapy only. Nine patients relapsed and eight died of disease. Six patients are currently alive and disease-free. In the whole series and particularly among these six survivors, neither age, gender, site of disease nor histology appeared to correlate with outcome. We find that the 
prognosis for pediatric GCTs containing TMSC is generally worse than that for GCTs alone. We also find that surgery plays an essential role in localized disease, with complete resection desirable. Chemotherapy chosen based on the histology of the malignant transformed component and on a clearly malignant GCT component, if present, is also valuable.

The role of surgery for localized disease in our series, especially with respect to patients \# 1, 3, and 5, is consistent with the conclusions of Biskup et al. [8] who studied a pediatric series of nine females (seven ovarian and two sacrococcygeal tumors). In that study, TMSC was always detected at diagnosis, no sarcomatous component was ever identified, while carcinoma was found in five cases. Six patients underwent surgery as a unique treatment (one sacrococcygeal and five ovarian tumors), and four of these patients are alive and disease-free without further therapies.

In adults, surgery remains the mainstay of treatment for TMSC in localized disease. Motzer et al. [3] report that the prognosis is more related to extent of disease and operability than to histological type, with complete resection of TMSC impacting favorably on survival. Donadio et al. [6] recommend surgery as the first choice of treatment in cases with a single tumor site. In the series of 14 adult males described by the French group [7], 3 of the patients where treated and cured with surgery only. Thus, in adults, it has been suggested that the surgeon be prepared to resect even adjacent organs or large vessels to ensure complete excision of the tumor [13]. In women, radical surgery, such as total hysterectomy, bilateral salpingooopherectomy, and lymphadenectomy, is associated with a better outcome [14].

In GCTs with TMCS, there are no typical clinical or radiological signs to predict malignant transformation of GCTs, which is one reason that surgery is advocated for patients with post-chemotherapy residual tumors or relapses, especially without evidence of elevated tumor markers [15-17]. Complete resection of residual masses after systemic therapy for GCTs might not only allow diagnosis of TMSC, but also prevent malignant transformation of residual teratoma. Note that there is no evidence that mature versus immature teratomas have more risk of malignant transformation [8]. The presentation of TMSC differs in different series, with $43 \%$ of adult cases identified within the primary tumor [3,7] and the remaining at the time of relapse [7] or in metastatic sites. By contrast, in the study by Biskup et al. [8], TMSC was already present at diagnosis in $100 \%$ of cases and in our series, in $78.5 \%$ of patients. In adults, it has been claimed that the transformation into adenocarcinoma takes longer than the other histologies $[6,18]$. In our series, all recurrences occurred within 2 years from diagnosis, including the case of adenocarcinoma. However, the carcinoma component is prevalent in females in the published series $[5,8]$ and in ours as well.

In advanced disease, survival may be improved by combining systemic therapy with loco- regional treatments. Surgery has been recommended for metastatic disease, depending also on the type of MSC detected, since a favorable impact on survival has been observed in all major published series $[5-7,18]$. It is worth nothing that the male (\# 14) with an MPNST component in retroperitoneal lymph node and lung metastases is disease-free after three surgical procedures involving the complete removal of the retroperitoneal lymph nodes and two subsequent thoracotomies.

Radiotherapy should be considered in addition to surgery, particularly when a sarcomatous component is identified. Although a clear-cut benefit has yet to be reported in adult with TMSC, radiotherapy has had a relevant clinical impact in selected cases of pediatric soft tissue sarcoma [19]. Of the nine patients in the present series who relapsed, only the patient (\# 7) given chemotherapy and radiotherapy focused on the rhabdomyosarcoma component is still alive and free of disease.

TMSC has been thought to be unresponsive to chemotherapy or less responsive to cisplatin-containing chemotherapy, but it is now known that the efficacy of chemotherapy depends on the histological type. In the presence of a malignant GCT type, cisplatin-based chemotherapy should be initiated to eradicate that part. Overall, GCT-directed chemotherapy is reasonable only if there is a clearly malignant GCT component (i.e.,YST, embryonal carcinoma, or choriocarcinoma). Donadio et al. [6] suggest that systemic therapy has a role in selected patients and that the expected response is based on histology, always requiring a multimodality approach to attempt eradication of all residual disease. In the 10 patients in our series who received chemotherapy at diagnosis, prognosis was improved when the chemotherapy was oriented to the transformed histology, consistent with reports in adults [6,7]. In fact, four patients still alive and disease-free (\# 5, 7, 12, and 14) received chemotherapy according to tumor cell type. Most exemplary of the benefit of this approach is patient \# 12 with stage IV neuroblastoma, who is alive after aggressive chemotherapy directed toward the somatic malignancy.

In published series and in ours as well, a few patients with complete resection underwent adjuvant chemotherapy; however, the total number of these cases is too small to draw conclusion about the usefulness of adjuvant therapy. It has been suggested that this choice should be dictated by type of transformed histology and whether adjuvant treatment has proven benefit in stage I disease of that histology [6].

Although conclusions from our retrospective study are limited by the small sample size, the heterogeneity of the patient series, and the lengthy time interval involved (29 years), we find a poor prognosis for GCTs associated with TMSC, consistent with reports in adults $[3,6,7]$. We also find that the optimal treatment was complete surgical resection of the tumor or resection of residual disease after systemic therapy. The role of adjuvant chemotherapy remains unclear. First-line therapy is helpful in advanced stages and should be optimized for histology. Chemotherapy should include reagents directed towards the somatic malignancy, if it is known to be chemosensitive disease. GCT-directed chemotherapy should be chosen only if there is a malignant GCT. Radiotherapy should be considered when TMSC is a sarcoma. Further large-scale cooperative efforts are warranted to enable clinical data collection and to perform biological studies.

\section{REFERENCES}

1. Ulbright TM, Loehrer PJ, Roth LM, et al. The development of nongerm cell malignancies within germ cell tumors: A clinicopathologic study of 11 cases. Cancer 1984;54:1824-1833.

2. Ahmed T, Bosl GJ, Haidu SI. Teratoma with malignant transformation in germ cell tumors in men. Cancer 1985;56:860-863.

3. Motzer RJ, Amsterdam A, Prieto V, et al. Teratoma with malignant transformation: Diverse malignant histologies arising in men with germ cell tumors. J Urol 1998;159:133-138.

4. Malagòn DH, Cano Valdez AM, Moran CA, et al. Germ cell tumors with sarcomatous components. A clinicopathologic and immnunoistochemical study of 46 cases. Am J Surg Pathol 2007;31:13561362. 
5. Rim SY, Kim SM, Choi HS. Malignant transformation of ovarian mature cystic teratoma. Int J Gynecol Cancer 2006;16:140-144.

6. Donadio CA, Motzer RJ, Dean F, et al. Chemotherapy for teratoma with malignant transformation. J Clin Oncol 2003;23:4285-4291.

7. El Mesbahi O, Terrier-Lacombe MJ, Rebischung C, et al. Chemotherapy in patients with teratoma with malignant transformation. Eur Urol 2007;51:1306-1312.

8. Biskup W, Calaminus G, Schneider DT, et al. Teratoma with malignant transformation: Experiences of the Cooperative GPOH Protocols MAKEI 83/86/89/96. Klin Padiatr 2006;218:303-308.

9. Corbett R, Carter R, MacVicar D, et al. Embryonal rhabdomyosarcoma arising in a germ cell tumor. Med Ped Oncol 1994; 23:497-592.

10. Cabay RJ, Setty S, Schwartz JL, et al. Pediatric squamous cell carcinoma arising in an alpha-fetoprotein-producing mature cystic teratoma of the mandible. Pediatr Blood Cancer 2009;52:130-132.

11. Cushing B, Giller R, Cullen JW, et al. Randomized comparison of combination chemotherapy with etoposide, bleomycin, and either high-dose or standard-dose cisplatin in children and adolescents with high-risk malignant germ cell tumours: A pediatric intergroup study-Pediatric Oncology Group 9049 and Children's Cancer Group 8882. J Clin Oncol 2004;22:2691-2700.
12. Michael H, Lucia J, Foster RS, et al. The pathology of late recurrence of testicular germ cell tumors. AM J Surg Pathol 2000;24:257-273.

13. Foster R. Editorial comment. Eur Urol 2007;51:1311.

14. Hacketal A, Brueggman D, Bohlmann MK, et al. Squamous-cell carcinoma in mature cystic teratoma of the ovary: Systemic review and analysis of published data. Lancet Oncol 2008;12:11731180 .

15. Droz JP. Editorial comment. Eur Urol 2007;51:1312.

16. Andre F, Fizazi K, Culine S, et al. The growing teratoma syndrome: Results of therapy and long-term follow up of 33 patients. Eur J Cancer 2000;36:1389-1394.

17. Sonnevald DJA, Sleijfer DTh, Schraffordt Koops H, et al. Mature teratoma identified after postchemotherapy surgery in patients with disseminated nonseminomatous testicular germ cell tumours. Cancer 1998;82:1343-1351.

18. George DW, Foster RS, Hromas RA, et al. Update on late relapse of germ cell tumor: A clinical and molecular analysis. J Clin Oncol 2003;21:113-122

19. Walterhouse D, Watson A. Optimal management for rhabdomyosarcoma in children. Paediatr Drugs 2007;9:391-400. 